\title{
The Study of Neutron Induced Reaction on Manganese Reaction Cross-Section at Intermediate Energy
}

\author{
Asmamaw Tsega Alemu*, Yenesew Asradew \\ Departments of Physics, College of Natural and Computational Science, Injibara University, Injibara, Ethiopia
}

Email address:

mulatetsega@gmail.com (A. T. Alemu)

${ }^{*}$ Corresponding author

\section{To cite this article:}

Asmamaw Tsega Alemu, Yenesew Asradew. The Study of Neutron Induced Reaction on Manganese Reaction Cross-Section at Intermediate Energy. Radiation Science and Technology. Vol. 7, No. 1, 2021, pp. 1-6. doi: 10.11648/j.rst.20210701.11

Received: December 3, 2020; Accepted: December 15, 2020; Published: January 12, 2021

\begin{abstract}
The general neutron induced reaction cross sections with the projectile neutron energy are being studied. As a result of this, the compound nucleus is formed through de-excitation of the selected nuclei by emitted particles $\gamma$ and $2 \mathrm{n}$ with the reaction cross section at intermediate energy. The production of residual nuclide by neutron induced reaction on the selected nuclei has been investigated by using computer code projection angular-momentum coupled evaporation Monte Carlo code (PACE4). Dependence of Reaction Cross Section for Projectile Neutron with Different K Values on Target 55Mn in the Energy Range (0.5 - 3.42) Mev and Dependence of Reaction Cross Section for Projectile Neutron with Different K Values on $55 \mathrm{Mn}$ Target in the Energy Range (11.5 - 38.5) Mev. Then, the theoretical estimated value compared with the Experimental value the data which obtained from International Atomic and Energy Agency, Exchange format. Results show us the reaction cross section vs energy of projectile neutron with target nucleus Manganese and the emitted particles were $\gamma$ and $2 \mathrm{n}$ respectively with new residual nuclei, i.e. $56 \mathrm{Mn}$ and $54 \mathrm{Mn}$, in addition to this the best fit $\mathrm{K}$ values are 10 and 8 for each channels respectively. Finally the level density parameter is the dominant parameter that can influence the reaction cross section from the outcome of this software by varying with free constant parameter (K). i.e., 8, 10 and 12 .
\end{abstract}

Keywords: Manganese, Level Density Parameter, Free Constant Parameter, Reaction Cross-section

\section{Introduction}

The investigation of characters of atomic nuclei and the fundamental physical relationships governing their interactions is the basis for all nuclear technologies. The process of yielding of new nuclei and elementary particles in collision of particles and nuclei is known as nuclear reaction. This is why the measurement and calculations of the cross section of nuclear reactions as much accurate as possible and understanding of nuclear reaction mechanisms is an important issue. The shape of the difference of reaction cross-section vs excitation energy reveals the reaction mechanisms [1].

For the purpose of study neutron interaction; neutrons can be classified in various categories. Depending up on their energy as:

1) Slow neutrons: are neutrons which have energy between 0 and 1000 ev

a) Cold neutrons: Neutrons having energy very small around 0:002ev. These neutrons have high penetrability in crystalline and poly-crystalline materials.

b) Thermal neutrons: Neutrons which they are in thermal equilibrium with the surroundings. The energy is not exactly known, but of the order of $\mathrm{E} \sim \mathrm{kT}$ if $\mathrm{T}=300 \mathrm{~K}$ and $\mathrm{k}=1.33 \times 10^{-16}$, then $\mathrm{E} \cong 0.025 \mathrm{ev}$.

c) Epithermal neutrons: are neutrons whose energy is greater than $0.5 \mathrm{ev}$.

d) Resonance neutron: are neutrons having energy between $1 \mathrm{ev}$ and $100 \mathrm{ev}$.

2) Intermediate neutrons: are neutrons which have energy $10^{3} \mathrm{ev}$ to $5 \times 10^{5} \mathrm{ev}$

3) Fast neutrons: neutrons which have energy range 0:5Mev to10Mev.

4) Very fast neutron: neutrons having energy range of $10 \mathrm{Mev}$ to $50 \mathrm{Mev}$

5) Ultrafast neutrons: neutrons having energy more than $50 \mathrm{Mev}$.

The study of neutrons interaction with matter requires the knowledge or awareness of neutron energy spectrum. For 
various applications the spectrum is poorly known. All neutrons are fast by nature and lose energy by colliding elastically with atoms in their environment and then later being slowed down to thermal energies they are picked by the nuclei of the absorbing medium [2].

A nuclear reaction is usually expressed in compact form as $\mathrm{X}$ (x, y) Y. The bombarding particle may be a neutron, proton, deuteron, triton, alpha-particle or any other heavy ion. The emitted particles may be a nucleon, nucleons, a nucleus or gamma-radiations [3]. In compound nuclei formation reactions, on the reaction of ${ }_{25}^{55} \mathrm{Mn}$ and with neutron and proton incident is picked by the target nucleus and its energy is shared and reshared among the nucleons of the compound nucleus until it arrives a state of statistically equilibrium. After a time much longer than the time required by the projectile to cross or pass the nucleus, a nucleon or a two or more of nucleons near the surface may, by a statistical fluctuation, gain enough energy to escape, just as a molecule may evaporate from a heated drop of liquid [4]. In order a nuclear reaction might formed, the nucleons in the projectile particles must interact with the nucleus in the target nucleuses. Thus, the energy must be exceeding than the natural electromagnetic repulsions between the interaction partners; this energy barriers are Coulomb barriers. If the energy is below the barrier energy, the nuclei will bounce off each other repeatedly. The energy barrier, Ec, or coulomb barrier depends on the atomic numbers of incident particle, must interact with the nucleus in the target. The energy barrier, Ec, depends on the atomic numbers of the target nucleus $Z_{1}$ and the atomic number of the projectile nucleus $Z_{2}$ [5]. It can be obtained according to the following equation;

$$
\mathrm{E}_{\mathrm{c}}=\frac{1}{4 \pi \varepsilon_{0}} \frac{Z_{1} e Z_{2} e}{r}
$$

When Ec is coulomb barrier energy and its unit is $\mathrm{MeV}$ and $\mathrm{r}$ in $\mathrm{cm}$

$$
E c=1.44 \times 10^{-13} \cdot \frac{Z 1 . Z 2}{r}-
$$

Where $r$ expressed the separation between the two nucleons and within this distance nuclear

Forces becomes operative. It can be formulated as follows;

$$
\mathrm{r}=\mathrm{r}_{0}\left(\mathrm{~A}_{1}{ }_{1 / 3}+\mathrm{A}_{2}^{1 / 3}\right)
$$

Where, $A_{1}$ is mass of projectile and A2, mass of target.

Coulomb Barrier Potential

Every nucleon surrounded by an electrostatic potential that opposes the entry and escape of the positively charged particles (proton, alpha, deuteron, etc.). Neutrons which are neutral particles are not faced coulomb barrier and accordingly are more readily absorbed and emitted by nuclei than are charged particles [6]. The coulomb barrier also impedes the release of a charged particle from the excited Compound nucleus. Also, the probability or cross section slow down steadily with increasing neutron energy, but in a low energy regions, which including the thermal range $(\mathrm{E}<1 \mathrm{eV})$. In this area, the absorption cross section, which is often has peak values, is inversely proportional to the velocity $\mathrm{v}$. This region is commonly referred to as the $1 / V$ region", since the absorption cross-section is proportional to $1 / \mathrm{v}$, which is the reciprocal of neutron velocity [7]. Consider a spherical target (outlined in grey in the figure 1) and a beam of particles (in blue) "flying" at speed v (vector in black) in the dimension of the target. We need to know how many particles impact it during time interval $\mathrm{dt}$. To obtain it, the projectiles have to be in the black cylinder in the figure 1 (volume V). The base of the cylinder is the geometrical cross section of the target orthogonal to the beam (surface $\sigma$ in red) and its height the length travelled by the particles during dt (length vdt) [8]:
Volume $=\sigma$ vdt

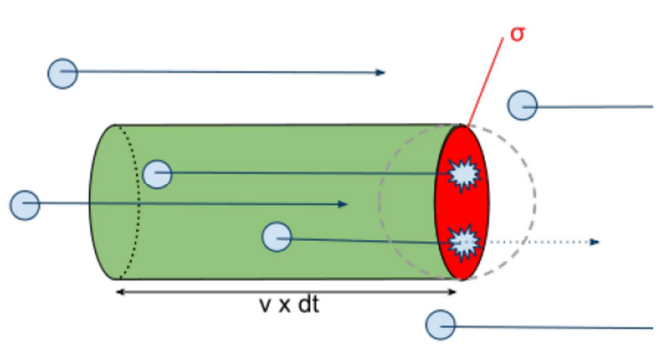

Figure 1. Interpretation of the reaction rate with the help of the nuclear cross section and projectile energy.

\section{Methodology}

\subsection{Computer Code and Formulation}

$\mathrm{T}$ do such kind of research it may use different code among this Pace 4 has available on my current status instead of others.

\subsection{PACE4 Code}

The PACE4 (projection angular-momentum coupled evaporation Monte Carlo code) this code is guided by a statistical approach. Within this program the de-excitation of the compound nucleus is cascaded by a Monte-Carlo procedure. The angular momentum projections are calculated at each level of de-excitation, which able to the de-excitation of the angular distribution of the released particles. The level density parameter is a basic parameter which may be varied to much the experimental data. The impact of variation in the (fitting parameter) $\mathrm{K}$ on calculated excitation functions for the selected nuclei varies the level parameter density to obtain fitted value with experimental [9]. The permitted maximum energy for this code is work properly below $2000 \mathrm{Mev}$.

$$
\text { Little- } \mathrm{A}=\mathrm{Acn} / \mathrm{K}
$$

The partial cross-sections $\left(\sigma_{1}\right)$ for Compound nucleus happened at angular momentum and

Specific bombarding energy is given by:

$$
\sigma_{1}=\frac{\pi \lambda^{2}}{4 \pi^{2}}(2 \mathrm{~L}+1) \mathrm{T}_{1}
$$

Where, $\lambda^{\prime}$ is the reduced wavelength and T1, the transmission coefficient given by

$$
\mathrm{T} 1=\left[1+\exp \left(\frac{l-l \max }{\Delta}\right)\right]^{-1},
$$


Where, $\Delta$ is the diffuseness parameter and lmax is determined by the total fusion cross-section of, since,

$$
\sigma \mathrm{f}=\sum_{l=0}^{\infty} \sigma \mathrm{l}
$$

It may be screen out that code PACE4 works only the statistical equilibrium model that is the formation of compound nucleus calculations and it does not concern for pre-equilibrium (PE) and in-complete fusion processes into consideration.

\section{Results and Discussions}

This chapter explains sample description of the analysis of the reaction result and, which are discussions of the main trends, patterns and connections that have emerged which is expressed by plotted graph. The cross section of neutron induced reaction on the new residue of $56_{\mathrm{Mn}}$ and $54_{\mathrm{Mn}}$ can be obtained from the excitation of ${ }_{25}^{56} \mathrm{Mn}^{*}$ for each reaction that we perform from Pace 4.

\subsection{Results}

\subsubsection{Channel 1}

Dependence of Reaction Cross Section for Projectile Neutron with Different $K$ Values on Target $55_{\mathrm{Mn}}$ in the Energy Range (0.5 - 3.42) Mev

From Figure 2, channel shows that the reaction of ${ }_{25}^{55} M n(n, \gamma){ }_{25}^{56} M n$, as a result of this gamma is ejected with bombarding energies from energy range of $0.5-3.42 \mathrm{Mev}$ this graph is plotted for both the theoretical obtained from PACE4 and experimental from IAEA, EXFOR library. In addition to this, from figure 3 , the formation of ${ }_{25}^{56} \mathrm{Mn}$ is obtained after excitation of ${ }_{25}^{56} \mathrm{Mn}^{*}$. Hence, the graph is better fitted from energy range $0.5-2.22 \mathrm{Mev}$, but beyond 2.22 Mev the formation of this radionuclide could not occurred, but the most fitted value for this graph is $\mathrm{K}=10$. However, neutron is penetrated with zero ( 0 ) target barrier height, since neutron is charge less light ion particle, it can penetrate easily the target nucleus with fusion radius $8.70 \mathrm{fm}$. The numbers of cascades are 1000 for the formation this reaction, but PACE4 can work less than 1000000 numbers of cascades; this reaction is better fitted with neutron energy below $2.22 \mathrm{Mev}$.

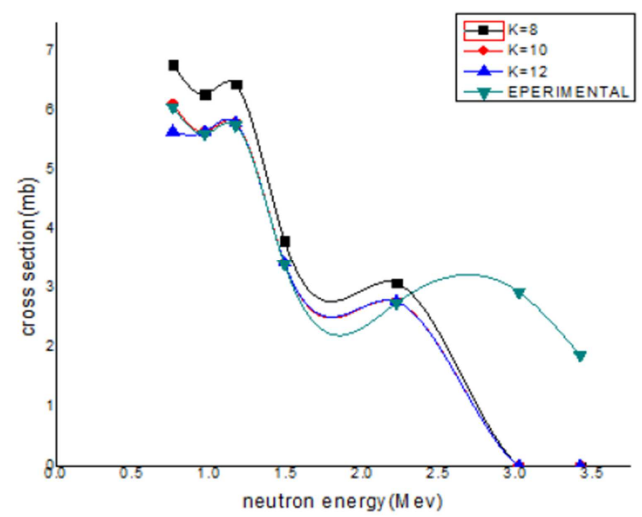

Figure 2. The influences of $K$ (free constant) in reaction cross section for residue ${ }_{25}^{56} \mathrm{Mn}$.

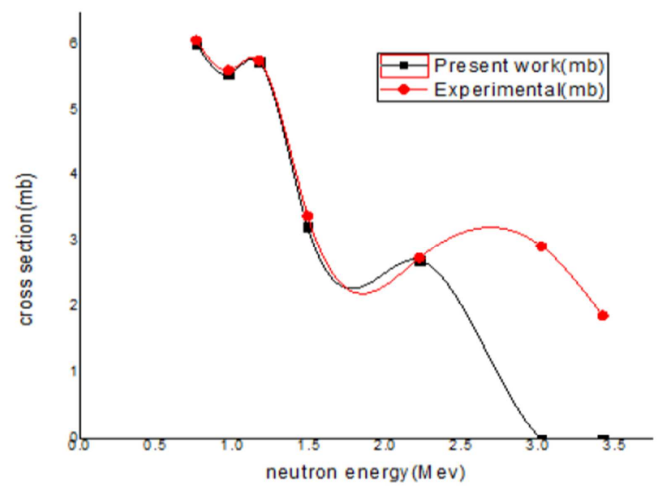

Figure 3. The best fit neutron induced reaction cross-section from the above figure 2. i.e, ${ }_{25}^{55} \operatorname{Mn}(n, \gamma){ }_{25}^{56} \mathrm{Mn},[10]$.

\subsubsection{Channel 2}

Dependence of Reaction Cross Section for Projectile Neutron with Different $\mathrm{K}$ Values on $55_{\mathrm{Mn}}$ Target in the Energy Range (11.5 - 38.5) Mev.

In Figure 4, it shows that the reaction of ${ }_{25}^{55} M n(n, 2 n)_{25}^{54} M n$, here is an ejected two neutron with bombarding neutron energies from the energy range $11.5-38.5$ Mev with different $\mathrm{K}$ values i.e 8, 10 and 12 , however, the formation of ${ }_{25}^{54} \mathrm{Mn}$, is obtained after excitation of ${ }_{25}^{56} \mathrm{Mn} *$, from figure 5 shows us almost a best fit to the experimental value in the shape of curve using free fit parameter constant $(\mathrm{K})=8$, but it can penetrate easily the target nucleus with fusion radius $8.70 \mathrm{fm}$, the number of cascade is 1000 for the formation of the events.

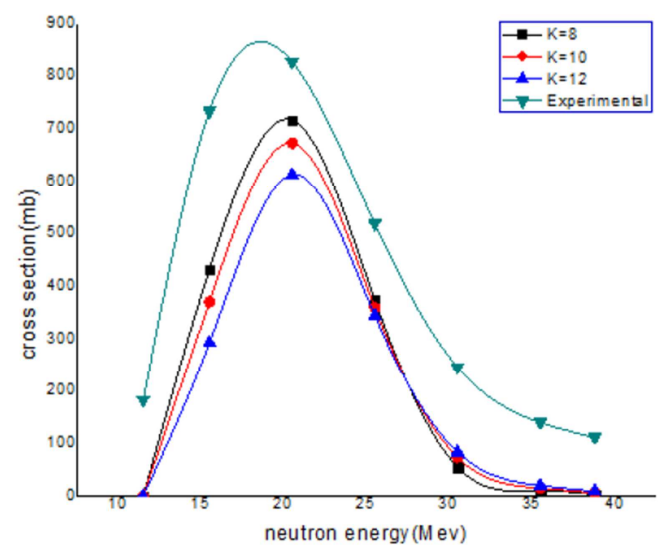

Figure 4. The influences of K interaction cross section for residue ${ }_{25}^{54} \mathrm{Mn}$.

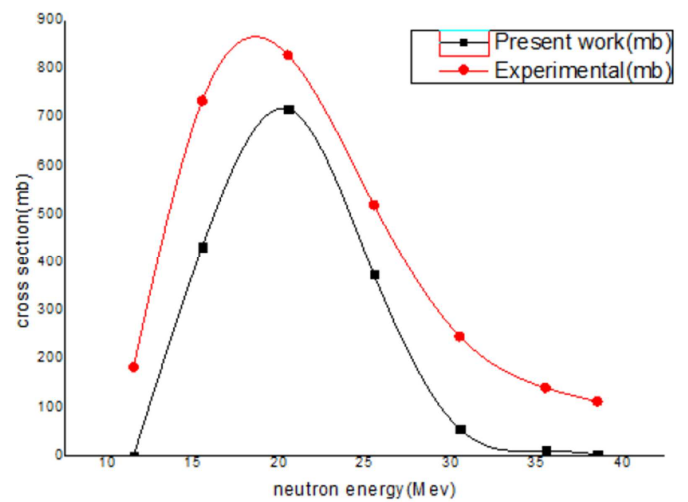

Figure 5. The best fit neutron induced reaction cross-section from the above figure ${ }_{25}^{55} \operatorname{Mn}(n, 2 n){ }_{25}^{54} \mathrm{Mn}$, [11]. 
From JANIS web site it describe the formation of new residues with cross section value supported by various models to arrive mean value, from the figure 6 and figure 7 we can compare and contrast this work with another models.

From figure 6 below, the energy range between $X_{\min }-X_{\max }$
$(10 \mu \mathrm{ev}-36 \mathrm{ev})$ and the cross section range $\mathrm{Y}_{\min }-\mathrm{Y}_{\max }$ (3.3594nb-7189.289b) [15], from this we can discussed that they are make a resonance structure with in the energy range (0.5-3.0) Mev. While beyond this energy this work (PACE4) cannot agree with the rest models.

\section{Mn55 $(n, y)$ or Mn56 production}
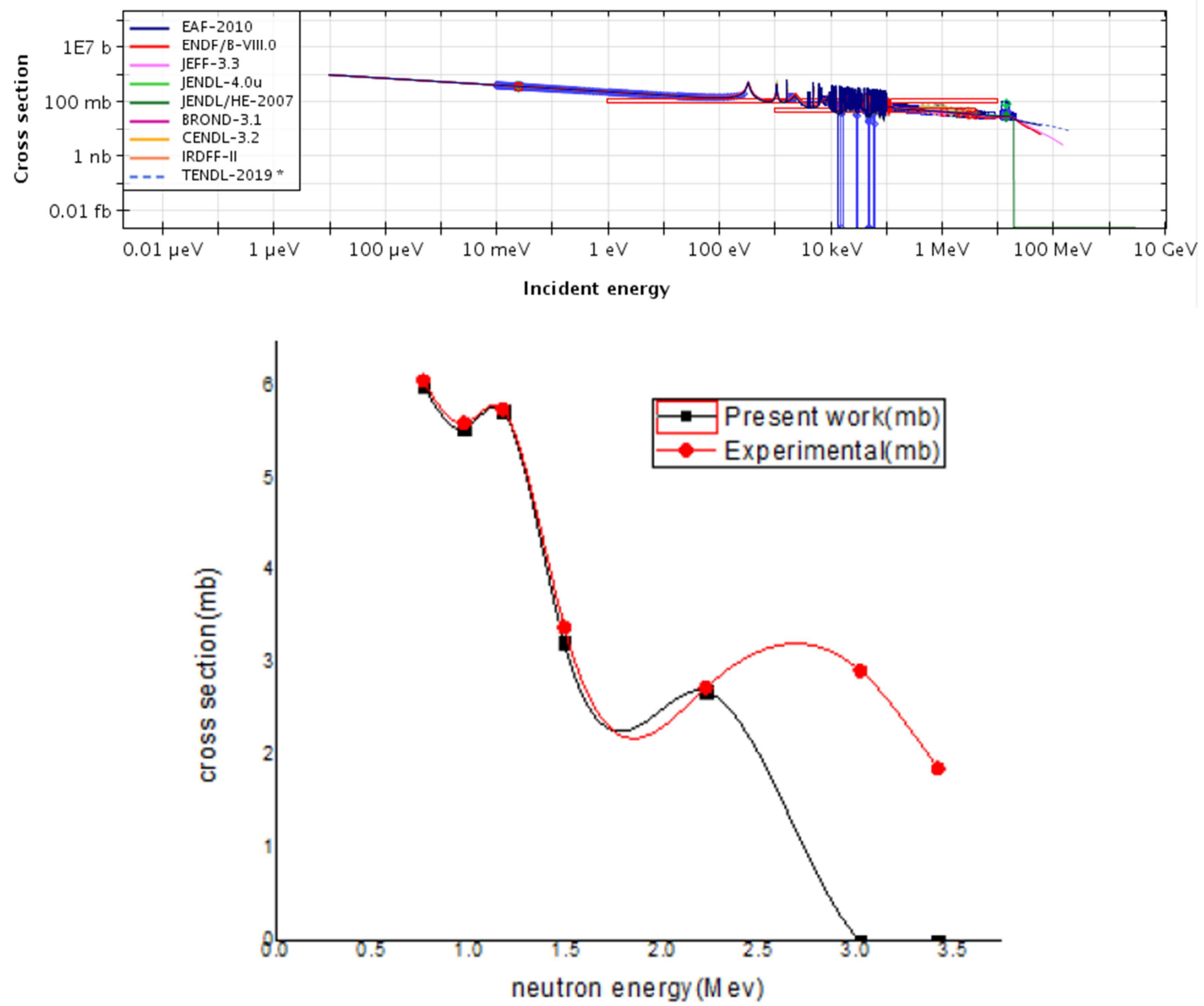

Figure 6. The various model can alter neutron induced reaction cross-section of ${ }_{25}^{55} \mathrm{Mn}(n, \gamma){ }_{25}^{56} \mathrm{Mn}$, and present work at the right side.

From Figure 7 below, it describes that different software or models can vary the values of cross sections of new residues with projectile neutron within the energy range $X_{\min }-X_{\max }$ (1Mev-3Gev) and cross section range between $\mathrm{Y}_{\min }-\mathrm{Y}_{\max }$ $(43.8947 \mu b-3.3425 b)$ [16]. From the two figure we can discussed as they are make a Gaussian structure, further they have good agreement with energy gap i.e from around (10 Mev $35 \mathrm{Mev}$ ) increasing the cross section has slight variation, but both of them are increasing up to peak value $20 \mathrm{Mev}$ then return to decreasing the value of cross section up to $35 \mathrm{Mev}$.

\section{Mn55 (n,2n) or Mn54 production}

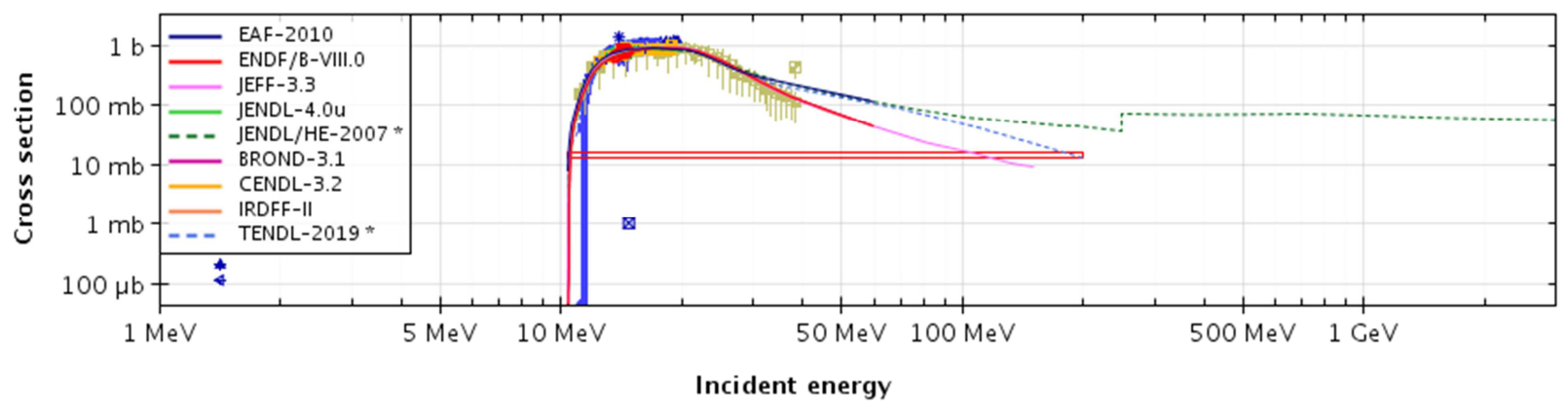




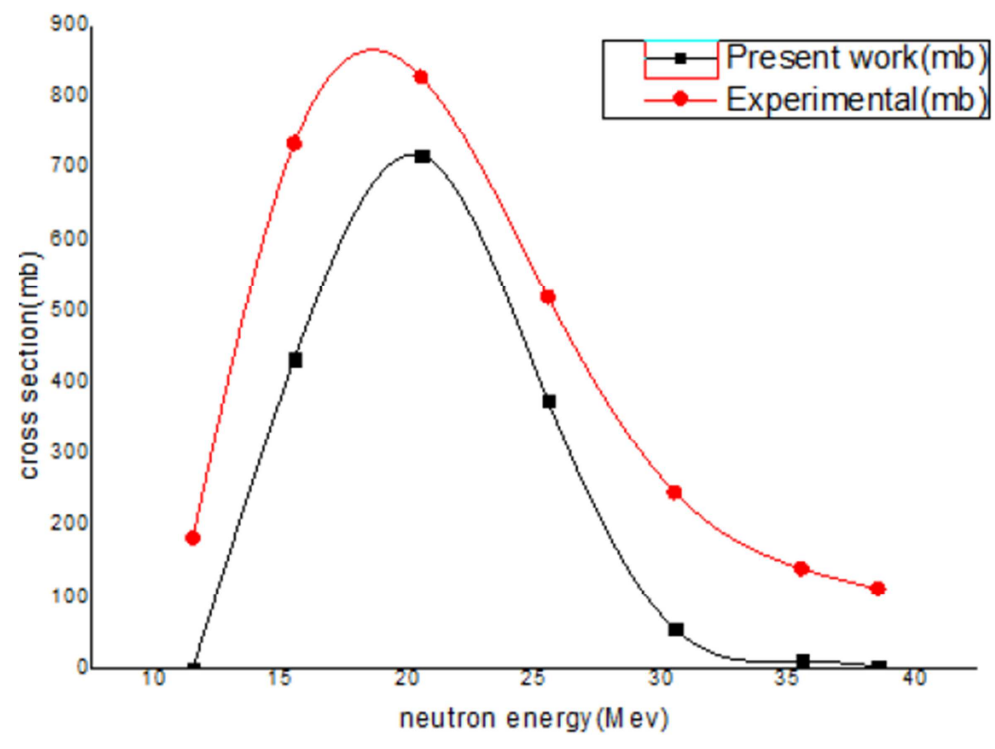

Figure 7. The various model can alter neutron induced reaction cross-section values of ${ }_{25}^{55} \mathrm{Mn}(n, 2 n){ }_{25}^{54} \mathrm{Mn}$, and present work at the right.

\subsection{Discussions}

From the above result, we see the output emitted particles are $\gamma, 2 \mathrm{n}$ and each channels has its own reaction cross section values which as supported by the following expressions.

The neutron-induced reaction cross sections have direct applications in calculating radiation levels as well as decay heat of various materials that have been exposed to radiation fields with strong neutron component [12].

The formerly-mentioned needs of complete and reliable neutron-induced reaction cross section data for $\mathrm{Mn}$ and $\mathrm{Cu}$ also enabled a stringent test of the differnt nuclear models and their corresponding account of specified effects. However, the main points in this respect are related to the Pre-equilbrum description which becomes increasingly significant at higher energies [13], but from this project mainly regarding on the occurrences of compound nucleus. In the excited compound nucleus, the de-excitation process includes obviously light particle emissions, $\gamma$-ray emissions, and fission. However, because of the existence of the Coulomb barrier for charged-particle emissions, the probability for the escaping of light charged particles is much smaller than the one for the neutron emission. As a result of this, most of the super heavy nuclei are obtained through the consecutive neutron evaporations [14].

\section{Conclusion}

The present work, the neutron induced reaction cross section on Manganese at intermediate energy are almost discussed. As a result of this, the compound nucleus is formed through de-excitation of the selected nuclei by emitted particles such as $\gamma, 2 \mathrm{n}$, using fitting parameter $(\mathrm{K})$ and little-A (level density parameter) are much influence the reaction cross section. Reaction cross section for channel one make a resonant structure between the energy range of $(0.5$ 2.2) Mev while, beyond this the cross section is down ward to zero, when we come to channel 2 or from figure 5 within the energy range of (11.5-36) $\mathrm{Mev}$ at $\mathrm{K}=8$, beyond this energy of neutron the cross section down to zero with a Gaussian shape. Indeed, the level density parameter is dependence of the free constant $\mathrm{K}$ are discussed using three possibility $\frac{A c n}{8}, \frac{A c n}{10}$ and $\frac{A c n}{12}$ for each channels of reactions, also different software have different cross section values. In general it makes good agreement with $\mathrm{K}$ values 10 and 8 , as we see from figure 3 and figure 5 respectively.

\section{List of Abbreviations}

$A_{c n}$ - Atomic mass of compound nucleus, EC- Coulomb Energy, Fm-Fermi meter, IAEA, EXFOR- International Atomic Energy Agency exchange Format, mb- milibarn, Mev-Mega electric volt, PACE4- projection angularmomentum coupled evaporation Monte Carlo code.

\section{Declarations}

\section{Availability of Data and Materials}

The raw dataset in origin V. 6 format for this article is available from the author Asmamaw Tsega: mulatetsega@gmail.com. As per your reasonable request the dataset attached as a file at asem.

\section{Funding}

This work supported by Injibara University which were not involved in design of study, data collection, analysis and interpretation and there is no financial conflict between the authors and individual or institution.

\section{Authors' Contributions}

ATA conceived and designed the study. The Author done study validation, formal analysis, investigation resources, 
data curation, writing (original draft preparation), writing (review and editing), Visualization, funding acquisition.

\section{Acknowledgements}

Behind the screen of my successes there is one, that is the almighty God, who never let me alone in all my work. Next, I would like to express my sincere thanks to my friend Habtamu Dessie (MSc) for his guidance, supervision and contribution of valuable suggestions. His scientific excitement, integral view on research has made a deepest impression on me. Also I would like to thanks all members of department of physics in Injibara University and all my friends for providing, encouragement and support, next to my family.

\section{References}

[1] Ejigu Kebede, The study of $\alpha$ - particle induced reaction on $27-$ Al and 197-Au Nuclides Addis Ababa university, (2014).

[2] Abdu Ali, Neutron Physics, Addis Ababa, Ethiopia June, 2011

[3] Awoke Tadesse, The study of $\alpha_{-}$Particle induced reaction on gallium isotopes Addis ababa university, (2007).

[4] Ntumba Lobo, Study of $20_{\mathrm{Ne}}$ induced reaction in $59_{\mathrm{Co}}$, Incomplete and complete fusion. Addis Ababa university, (2016).

[5] Keller. C, Radiochemistry, Haleted Press, JOHN WILEY\&SON, (1988).
[6] Getanehayele, Study of proton induced reaction - cross section for various energies on yttrium and scandium, Addis Ababa university, (2007).

[7] http://nuclearpowertraining.tpub.com/h1019v1/css/Figure-1Typical-Neutron Absorption-Cross-Section-Vs-NeutronEnergy-113.htm.

[8] https://en.wikipedia.org/wiki/Neutron_cross_section.

[9] Biniyam Nigussie, Complete and incomplete fusion studies in some $14 \mathrm{~N}+59$ Co systems, Addis Ababa university, (2012).

[10] https://www.iaea.org/exfor/servlet/x4sgereacTablrepx. (auther) Y. Uwamino, H. supita, Y. kondo, T. Nakamurad.

[11] https://www-nds.iaea.org/exfor/servlet/. (auther) R. p. Guutam. Es. Al, (1992).

[12] https://www.oecdnea.org/janisweb/book/neutrons/Mn55/MT102/renderer/264.

[13] https://www.oecdnea.org/janisweb/book/neutrons/Mn55/MT16/renderer/277.

[14] P. M. Prajapati et al., Measurement of neutron-induced reaction cross-sections in zirconium isotopes at thermal, 2.45 $\mathrm{MeV}$ and 9.85 MeV energies, Nucl. Sci. Eng. 171, 78 (2012).

[15] "Horia Hulubei" National Institute for Physics and Nuclear Engineering, PO Box MG-6, 077125 Bucharest, Romania b V. G. Khlopin Radium Institute, 2nd Murinski Ave. 28, St. Petersburg 194021, Russia Fast-neutron induced preequilibrium reactions on $55 \mathrm{Mn}$ and $63,65 \mathrm{Cu}$ at energies up to $40 \mathrm{MeV}, 21$ March 2008.

[16] Ling Liu, Caiwan Shen, Qingfeng Li, Ya Tu, Xiaobao Wang, and Yongjia WangResidue cross sections of 50Ti-induced fusion reactions based on the two-step model. 